Published in final edited form as:

Urology. 2018 April ; 114: 41-44. doi:10.1016/j.urology.2018.01.013.

\title{
Emergency Department Switching and Duplicate Computed Tomography Scans in Patients with Kidney Stones
}

\author{
Parth K. Shah, MD, Phyllis L. Yan, MA, Casey A. Dauw, MD, Brent K. Hollenbeck, MD, MS, \\ Khurshid R. Ghani, MD, Amy N. Luckenbaugh, MD, and John M. Hollingsworth, MD, MS \\ Dow Division of Health Services Research, Department of Urology, University of Michigan \\ Medical School
}

\section{Abstract}

Objective-To test whether duplicate imaging relates to a lack of information sharing among providers, we measured the association between emergency department (ED) switching during a kidney stone episode and receipt of a repeat computed tomography (CT) scan.

Methods-Using the MarketScan Commercial Claims and Encounters Database, we identified adults between the ages 18 and 64 with an ED visit for a diagnosis of kidney stones. Among patients who had an abdominal/pelvic CT scan at their initial encounter, we then determined the subset that made an ED revisit within 30 days of their first, distinguishing between those to the same versus a different ED. Finally, we fit multivariable logistic regression models to estimate the risk of receiving a repeat CT scan associated with ED switching.

Results-Twelve percent of patients who received a CT scan at their initial ED encounter had a revisit within 30 days of discharge. One third of their revisits were made to a different ED than the index one. Duplicate CT scans were obtained at nearly $40 \%$ of all revisits. On multivariable analysis, the risk of receiving a repeat CT was $12 \%$ higher if this revisit was made to a different ED (risk ratio, $1.12 ; 95 \%$ confidence interval, 1.03 to $1.21 ; P=0.010$ ).

Conclusions-Our study reveals that ED switching during an acute kidney stone episode is associated with higher levels of repeat CT imaging. These findings support the role of better health information exchange among providers in order to help reduce waste in the healthcare system.

\section{Keywords}

Renal Colic/diagnostic imaging; Emergency Service; Hospital; Tomography; X-Ray Computed/ utilization

\section{Introduction}

Expenditures made by Americans on healthcare total $\$ 3.3$ trillion annually, accounting for $17.9 \%$ of the gross domestic product. ${ }^{1}$ While healthcare spending growth has slowed

Corresponding author: John M. Hollingsworth, MD, MS, Associate Professor of Urology, University of Michigan Medical School, 2800 Plymouth Road, Building 16, $1^{\text {st }}$ Floor, Room 112W, Ann Arbor, MI 48109-2800, Tel: 734-763-2797, Fax: 734-232-2400, kinks@med.umich.edu.

Declaration of interest: None. 
significantly, the United States still pays more than any other nation in the world for healthcare. ${ }^{2}$ Forty to $50 \%$ of this spending is considered waste-a substantial portion of which can be explained by the high prevalence of duplicate (sometimes unnecessary) medical imaging. ${ }^{3}$ Thus, efforts to limit duplicate imaging may go a long way towards reducing healthcare spending. ${ }^{4}$

To limit duplicate imaging, a better understanding of its causes is needed. Known causes include patient demand, defensive medicine, and fee-for-service reimbursement. ${ }^{5}$ Another potential cause that has been incompletely explored relates to failures of care coordination. During an episode of care, patients must often visit multiple, sometimes competing, providers dispersed across locations over time. If information is not shared among these providers, they may fail to see the full clinical picture and inadvertently order duplicate imaging. ${ }^{6}$

In this context, we analyzed claims data from privately insured adults with an emergency department (ED) visit for kidney stones. Among those who underwent a computed tomography (CT) scan at their initial evaluation, we determined the subset that had an ED revisit within 30 days of their first. We then measured the frequency of CT use during these revisits, distinguishing between revisits to the same versus a different ED. Our study will provide clinician leaders with actionable insights as they refine systems for sharing information sharing across care locations.

\section{Material and Methods}

For our study, we used Truven Health Analytics' MarketScan Commercial Claims and Encounters Database (2003 to 2006). This database captures medical and pharmacy claims data from working age adults with employer-sponsored insurance and their dependents.

To begin, we used an International Classification of Diseases, Ninth Revision, Clinical Modification (ICD-9) diagnosis code-based algorithm to identify beneficiaries between the 18 and 64 years of age, for whom an ED claim for kidney stones was submitted on their behalf. ${ }^{7}$ Because we were interested in incident stone episodes, we required that beneficiaries had a clean 90-day window with no other claims for kidney stones prior to their index ED visit. To be able to follow beneficiaries over their stone episodes, we required continuous enrollment in a benefit plan for 30 days after their index ED visit.

Next, we used appropriate ICD-9 procedure codes and Current Procedural Terminology codes to determine which beneficiaries received a CT scan of the abdomen and/or pelvis during their index ED visit. Among these beneficiaries, we subsetted further to distinguish those with an ED revisit for kidney stones within 30 days of their index visit. We excluded those who underwent kidney stone surgery between their ED visits.

\section{Exposure assessment}

To differentiate between revisits to the same versus a different ED, we used unique facility identifiers (IDs) to assign each visit to the facility associated with the plurality of claims on the visit date in question. For ED visits resulting in a hospitalization, we considered claims 
between the admission and discharge dates, and for outpatient ED visits, we examined claims filed within a 24-hour window around the visit date. To remove noise from our data, we only excluded unique facility IDs that were linked to different ZIP codes during the study period.

\section{Outcome measures}

Our primary outcome was receipt of duplicate imaging. Consistent with the prior literature, 8,9 we said that a beneficiary received duplicate imaging if he/she underwent a CT scan on the same body part (i.e., abdomen and/or pelvis) at both his/her index and repeat ED visits.

Our secondary outcome was receipt of a duplicate narcotic prescription. To determine whether this occurred, we used appropriate National Drug Codes to identify prescription fills for narcotic analgesics at both the index and repeat ED visits. Given the existence of prescription drug monitoring programs, our a priori hypothesis was that the rate of duplicate narcotic prescription was less susceptible to ED switching. ${ }^{10}$

\section{Statistical analysis}

For our initial analytic step, we characterized imaging use at the index ED visit for kidney stones. Among those beneficiaries who received imaging, we classified the type: CT, X-ray radiography, ultrasonography (US), or intravenous pyelography (IVP).

For the subset of beneficiaries who underwent CT imaging and then had an ED revisit, we then made bivariate comparisons between those with visits to the same versus a different $\mathrm{ED}$, using parametric and nonparametric tests where appropriate. Specifically, we compared patients with respect to their age, gender, employee classification (salaried versus nonsalaried), employment status (full- versus part-time), benefit plan type (comprehensive, preferred provider organization, health maintenance organization, point-of-service plan, or other), urban versus rural location, level of comorbidity (using a modified version of the Charlson index ${ }^{11}$ ) and region of residence (Northeast, Midwest, South, or West).

Finally, we used multivariate logistic regression to estimate the association between ED switching and the receipt of duplicate imaging and narcotic prescription, controlling for those sociodemographic and comorbid disease factors described above. To account for the correlation in our data (patients nested within facilities), we calculated robust standards errors using the Huber-White sandwich estimator. ${ }^{12}$ We used methods described by Zhang and $\mathrm{Yu}$ to approximate a risk ratio (RR) from the adjusted odds ratio. ${ }^{13}$

We performed two-sided significance testing and set a type-I error rate at 0.05 . All analyses were done using SAS software, version 9.4 (SAS Institute Inc., Cary, NC). The University of Michigan's Health Sciences Institutional Review Board deemed that this study was exempt from its oversight.

\section{Results}

In total, we identified 166,639 beneficiaries with an ED visit for kidney stones over the study interval. More than $86 \%$ of them received some sort of medical imaging during their 
index visit. CT was used most commonly (77.9\% of visits), followed by X-ray (22.3\%), IVP $(13.6 \%)$, and US (4.2\%) (Figure 1). Narcotics were prescribed at almost half (49.1\%) of index visits.

Nearly one in seven (13.5\%) had an ED revisit for kidney stones within 30 days of their first. Seventy-one percent of them had CT imaging at their index visit. For the subset of these beneficiaries who did not undergo stone-directed surgery between visits and where there was reliable reporting of the ED facility IDs in their claims $(n=3,818)$, over one-third (36.5\%) presented to a different ED than the one where they were seen for their index visit.

Table 1 displays differences between patients who presented to the same versus a different ED. In general, patients who switched EDs tended to live in an urban area $(P=0.002)$, were more likely to be paid on an hourly basis $(P=0.018)$, have part-time employment $(P<0.001)$, and reside in the South West $(P<0.001)$.

Two in five beneficiaries with an ED revisit (39.7\%) received duplicate CT imaging. The rates of duplicate CT imaging in the study population (Figure 2) increased over time ( $P=0.050$ for the temporal trend). The overall rate of repeat narcotic prescription at an ED revisit was $39.1 \%$. This rate was stable throughout the study period $(P=0.677$ for the temporal trend).

As shown in Figure 3, the likelihood of receiving duplicate CT imaging was higher among beneficiaries who switched EDs even after accounting for measurable patient differences $(P=0.010)$. Put differently, beneficiaries seen at a different ED than the one they originally presented to had a $12 \%$ higher risk of receiving duplicate CT imaging [RR, 1.12; 95\% confidence interval (CI), 1.03 to 1.21]. No such association existed between ED switching and duplicate narcotic prescription (RR, $0.93 ; 95 \% \mathrm{CI}, 0.82$ to $1.04 ; P=0.203$ ). In a sensitivity analysis, where we extended the time window during which a patient could have no other claims for kidney stones prior to his/her index ED visit from 90 to 180 and 365 days, our findings were robust.

\section{Discussion}

Patients experiencing an acute kidney stone episode often present to EDs, where CT imaging is frequently used to guide management. Our study demonstrates that a substantial proportion of these patients have an ED revisit within 30 days of their initial encounter, over a third of which are to different EDs. This ED switching is associated with a 12\% higher risk of a duplicate (and potentially unnecessary) scan. These findings have implications on efforts to reduce healthcare spending, highlighting the importance of care coordination and information sharing between providers.

Our results are consistent with those from prior studies on hospital switching among patients with other chronic conditions marked by acute exacerbations. For patients with epilepsy and chronic back pain, hospital switching has been shown to be associated with greater use of healthcare services, including imaging. ${ }^{14}$ In addition, empirical work on patients with congestive heart failure has demonstrated that hospital switching leads to significantly higher rates of duplicate medical procedures. ${ }^{15}$ One proposed explanation for this 
phenomenon relates to the difficulty of transferring clinical data between nonaffiliated sites. To expedite the patient evaluation, providers may choose to repeat testing. ${ }^{15}$

Along these lines, our findings on the lack of an association between ED switching and duplicate narcotic prescription have face validity. Most states have launched prescription monitoring programs, which are designed to enhance information sharing between providers and reduce the over prescribing of controlled substances. In fact, many studies from the emergency medicine literature have demonstrated a drastic reduction in rates of duplicate opioid prescriptions after the implementation of these programs. ${ }^{16}$

Our study must be considered in the context of several limitations. First, our analysis is limited by the accuracy of medical claims data. That being said, the diagnosis code-based algorithm that we used has been previously validated, demonstrating high specificity in identifying patients with urinary stone disease. ${ }^{7}$ Second, the Marketscan database is a convenience sample that includes only insured and employed individuals; however, this is the precisely the population that is most affected by kidney stones. The third limitation pertains to the recency of our data. The Health Information Technology for Economic and Clinical Health Act was signed into law in 2009, which led to a large-scale increase in the amount of health information recorded electronically. Despite this increase, duplicate imaging likely persists due to the fact that meaningful use of and interoperability between electronic health record systems are lacking. ${ }^{17}$

Notwithstanding these limitations, our findings have important implications for policymakers and patients. It is estimated that $\$ 20$ billion in healthcare spending is attributable to duplicate imaging due, in part, to communication failures between providers.

18 As such, there is an immediate need for the implementation of more effective health information exchange. This could be accomplished through greater hospital participation in region-wide health information organizations. These are third-party organizations that serve as hubs to all providers in a geographic area and help facilitate crosstalk between nonaffiliated health systems. ${ }^{19}$

From the patient perspective, our findings have implications on safety. Although low-dose protocols exist, ${ }^{20} 21 \mathrm{CT}$ scans remain the largest source of medically-related ionizing radiation exposure in the United States. ${ }^{21}$ Additionally, the overall rate of CT use has increased over the past two decades. ${ }^{22}$ This increase is thought to have contributed to a substantial number of incident cancer diagnoses. ${ }^{23}$ Therefore, insofar as more effective health information exchange reduces the number of duplicate CT scans obtained, patient safety would improve.

\section{Conclusions}

In summary, ED switching during an acute stone episode was associated with higher rates of duplicate CT imaging. These findings highlight the need for more effective health information exchange between health systems. 


\section{Acknowledgments}

Funding support: This study was supported by the National Institutes on Aging (R01AG048071 to BKH) and the Agency for Healthcare Research and Quality (1R01HS024525 01A1 and 1R01 HS024728 01 to JMH).

\section{References}

1. Centers for Medicare and Medicaid Services. National Health Expenditures 2016 Highlights. Available at: https://www.cms.gov/Research-Statistics-Data-and-Systems/Statistics-Trends-andReports/NationalHealthExpendData/downloads/highlights.pdf. Accessed on January 4, 2018

2. Keehan SP, Sisko AM, Truffer CJ, et al. National health spending projections through 2020: Economic recovery and reform drive faster spending growth. Health Aff (Millwood). 2011; 30:1594. [PubMed: 21798885]

3. Berwick DM, Hackbarth AD. Eliminating waste in U.S. healthcare. JAMA. 2012; 30:1513.

4. Bailey JE, Pope RA, Elliott EC, et al. Health information exchange reduces repeated diagnostic imaging for back pain. Ann Emerg Med. 2013; 62:25. [PubMed: 23711879]

5. Delaune, J., Everett, W. Waste and Inefficiency in the US Healthcare System. Cambridge, MA: New England Healthcare Institute; 2008.

6. Stiell A, Forster A, Stiell IG, et al. Prevalence of information gaps in the emergency department and the effect on patient outcomes. CMAJ. 2003; 196:1023.

7. Semins MJ, Trock BJ, Matlaga BR. Validity of administrative coding in identifying patients with upper urinary tract calculi. J Urol. 2010; 184:190. [PubMed: 20478584]

8. Lammers EJ, Alder-Milstein J, Kocher KE. Does health information exchange reduce redundant imaging? Evidence from emergency departments. Med Care. 2014; 52:227. [PubMed: 24374414]

9. Kamat AB, Midgley S, Kimbrell K. Duplication of radiology imaging studies in the emergency department: What is the cost? Emerg Med J. 2015; 32:144. [PubMed: 24096859]

10. Bao Y, Pan Y, Taylor A, et al. Prescription drug monitoring programs are associated with sustained reductions in opioid prescribing by physicians. Health Aff (Millwood). 2016; 36:1045.

11. Charlson ME, Pompei P, Ales KL, et al. A new method of classifying prognostic comorbidity in longitudinal studies: development and validation. J Chronic Dis. 1987; 40:33.

12. Williams RL. A note on robust variance estimation for cluster-correlated data. Biometrics. 2000; 56:645. [PubMed: 10877330]

13. Zhang J, Yu KF. What's the relative risk? A method of correcting the odds ratio in cohort studies of common outcomes. JAMA. 1998; 280:1690. [PubMed: 9832001]

14. Grinspan ZM, Abramson EL, Banerjee S, et al. People with epilepsy who use multiple hospitals; prevalence and associated factors assessed with health information exchange. Epilepsia. 2014; 55:734. [PubMed: 24598038]

15. Ayabakan, S., Zheng, Z., Bardhan, IR., et al. Value of health information sharing in reducing healthcare waste: an analysis of duplicate testing across hospitals. Thirty Fifth International Conference on Information Systems; Auckland. 2014. Available at: https:// pdfs.semanticscholar.org/8b95/dc6a2970e0b61bb56f5bd560b4956aef34c7.pdf. Accessed on January 4, 2018

16. Neven D, Paulozzi L, McPherson S, et al. A randomized controlled trial of a citywide emergency department care coordination program to reduce prescription opioid related emergency department visits. J Emerg Med. 2016; 51:498. [PubMed: 27624507]

17. Adler-Milstein J, Jha AK. Health information exchange among U.S. hospitals: Who's in, who's out, and why? Healthc (Amst). 2014; 2:26. [PubMed: 26250086]

18. Iglehart JK. Heath insurers and medical imaging policy: A work in progress. N Engl J Med. 2009; 360:1030. [PubMed: 19264694]

19. Hillestad R, Bieglow J, Bower A, et al. Can electronic medical record systems transform health care? Potential health benefits, savings, and costs. Health Aff (Millwood). 2005; 24:1103.

[PubMed: 16162551] 
20. Jellison FC, Smith JC, Heldt JP, et al. Effect of low dose radiation computerized tomography protocols on distal ureteral calculus detection. J Urol. 2009; 182:2762. [PubMed: 19837431]

21. Larson DB, Johnson LW, Schnell BM, et al. National trends in CT use in the emergency department: 1995-2007. Radiology. 2011; 258:164. [PubMed: 21115875]

22. Smith-Bindman R, Miglioretti DL, Johnson E, et al. Use of diagnosing imaging studies and associated radiation exposure for patients enrolled in large integrated health care systems, 1996-2010. JAMA. 2012; 307:2400. [PubMed: 22692172]

23. Brenner DJ, Hall EJ. Computed tomography-An increasing source of radiation exposure. N Engl J Med. 2007; 357:2277. [PubMed: 18046031] 


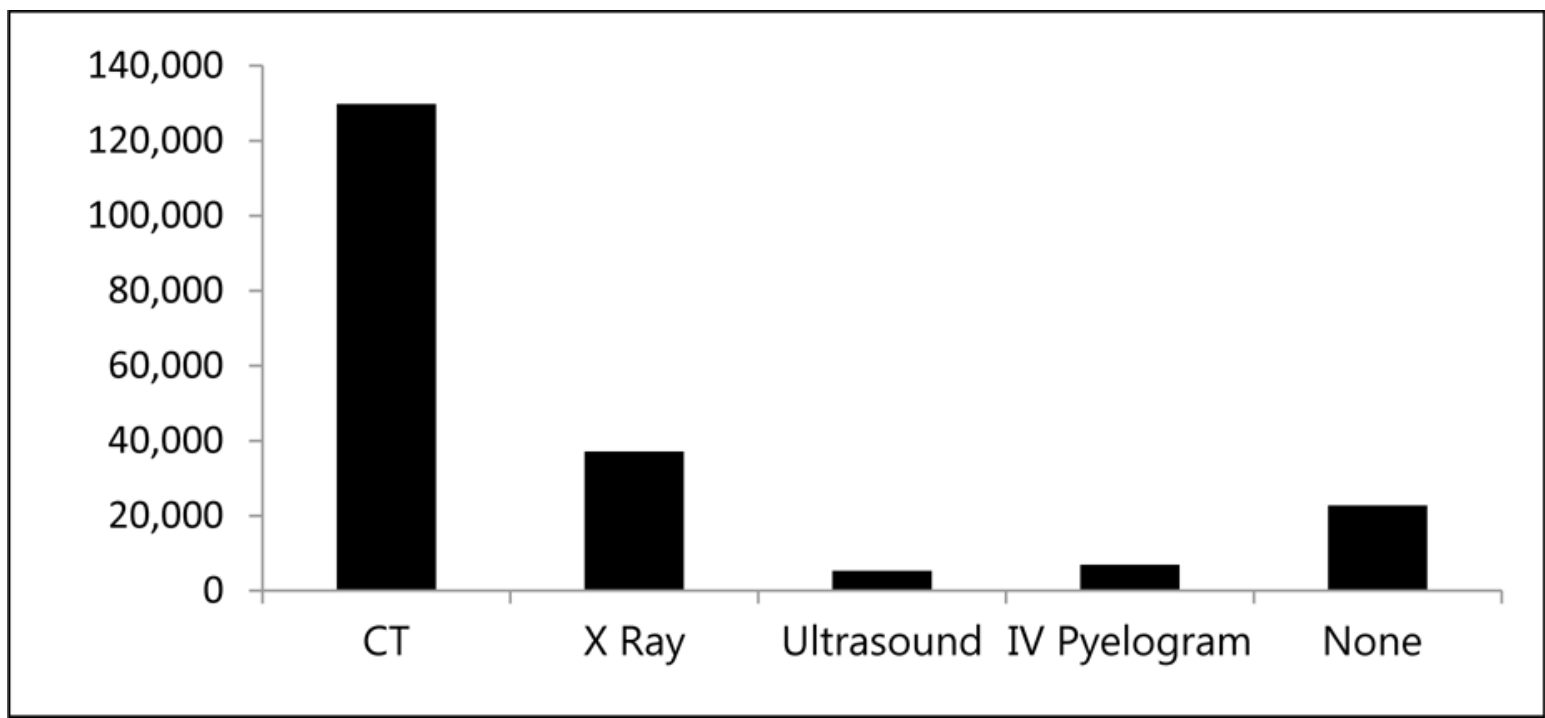

Figure 1.

Types of imaging studies obtained at the index ED visit.

Abbreviations: CT, computed tomography; IV, intravenous. 


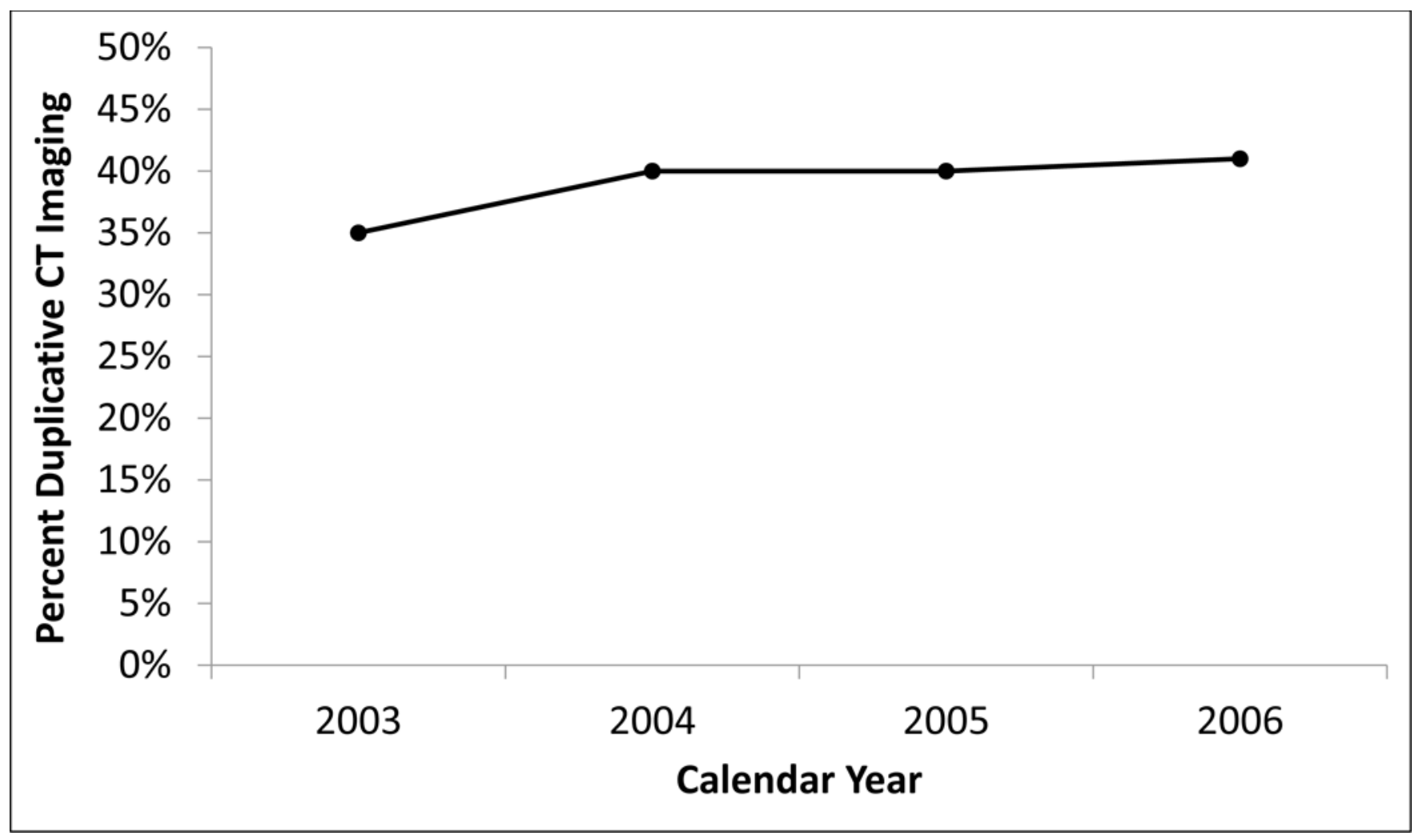

Figure 2.

Temporal trends in the percentage of ED revisits resulting in duplicate CT imaging. Abbreviations: CT, computed tomography; ED, emergency department. 


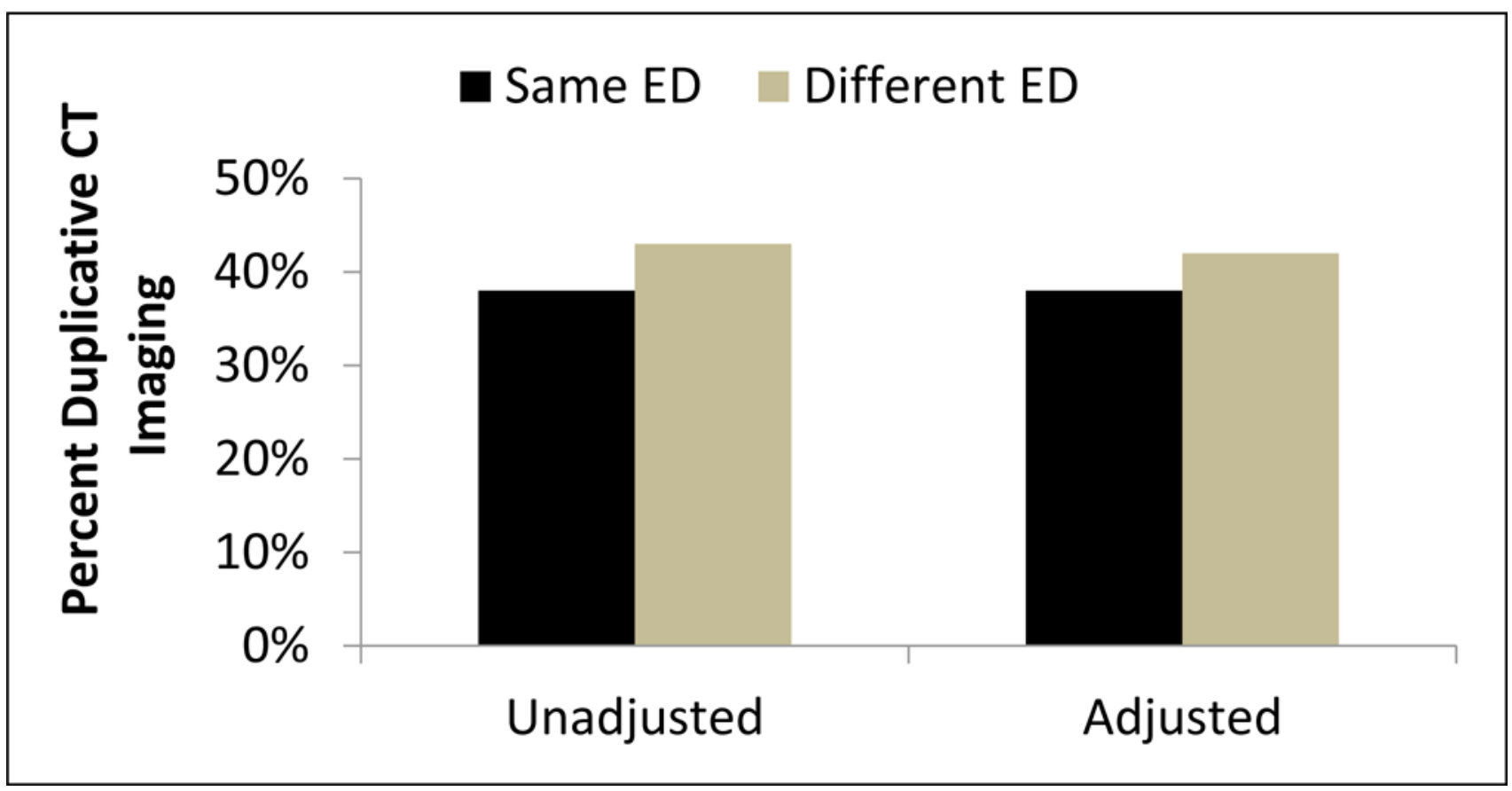

Figure 3.

Probability of duplicate CT imaging, same versus different ED.

Abbreviations: CT, computed tomography; ED, emergency department. 
Table 1

Comparisons between patients that had revisits to the same ED versus a different ED.

\begin{tabular}{|c|c|c|c|}
\hline & Same ED & Different ED & $P$-Value \\
\hline Age & & & 0.262 \\
\hline Age $\leq 30$ years & 16.4 & 17.8 & \\
\hline Age $>30$ years & 83.6 & 82.2 & \\
\hline Employment Class & & & 0.018 \\
\hline Salaried & 12.1 & 9.6 & \\
\hline Nonsalaried & 87.9 & 90.4 & \\
\hline Employment Status & & & $<0.001$ \\
\hline Full-time & 49.8 & 42.5 & \\
\hline Part-time & 50.2 & 57.5 & \\
\hline Charlson score & & & 0.005 \\
\hline 0 & 87.1 & 89.8 & \\
\hline 1 & 9.9 & 6.7 & \\
\hline 2 & 1.9 & 2.4 & \\
\hline$\geq 3$ & 1.2 & 1.1 & \\
\hline Locale & & & 0.002 \\
\hline Rural & 23.9 & 19.4 & \\
\hline Urban & 76.1 & 80.6 & \\
\hline Plan Type & & & $<0.001$ \\
\hline HMO & 19.1 & 25.6 & \\
\hline NonHMO & 80.9 & 74.4 & \\
\hline Region & & & $<0.001$ \\
\hline Midwest & 30.9 & 21.0 & \\
\hline Northeast & 10.7 & 5.4 & \\
\hline Southern & 44.8 & 56.6 & \\
\hline Western & 13.6 & 17.0 & \\
\hline Sex & & & 0.608 \\
\hline Female & 34.1 & 34.9 & \\
\hline Male & 65.9 & 65.1 & \\
\hline
\end{tabular}

Abbreviations: ED, emergency department; HMO, health maintenance organization. 